\title{
Practical Power System Aggregation Considering Dynamic Loads
}

\author{
Yoshio Yamagishi Member (Hokuriku Electric Power Company, yamagishi.yoshio@ rikuden.co.jp) \\ Shintaro Komami Member (Hokuriku Electric Power Company, komami.shintaroh@rikuden.co.jp)
}

Keywords: power system aggregation, power system stability, dynamic load model, generating unit model, excitation system

This paper describes Y-connection method for creating power system equivalent networks to be used in power system stability analysis, shown in Fig. 1(a). The Y-connection method, which is a type of coherency method, has the following feature:

- It uses three branches to connect the boundary bus, generator and load.

- Dynamic loads and distributed generators, which are difficult for eigenvalue methods to model, can be easily modeled.

- It can take into account reactance from the boundary bus to a load and a distributed generator. The accuracy of simulations is improved by considering that reactance.

In the Y-connection model three conditions are claimed.

(1) Transient impedance of the subsystem observed from trunk system is preserved, i.e., short-circuit current from the subsystem to trunk system is preserved.

(2) Output $\mathrm{kW}$-weighted average angle of generators is preserved. Therefore stability of generators may be preserved.

(3) Demand $\mathrm{kW}$-weighted average load angle is preserved. Therefore stability of loads may be preserved.

There are three unknown impedances and three conditions, therefore, the three impedances are calculated separately. In the Yconnection model, real and reactive power losses are approximately preserved.

The preservation can be proved using a "complex angle" $\theta$, which is defined as the summation of the product of impedance $\mathrm{Z}$ and power flow $\mathrm{P}$, from boundary bus to the point in question as follows:

$$
\theta=\sum(Z P)
$$

This paper also describes a practical procedure to determine the parameters used in the coherency method. These include the aggregated generator, excitation system, PSS (power system stabilizer), and speed governors. In aggregating the excitation system, the weighted average of frequency response and forcing ability are preserved. Forcing ability is evaluated by rise time and ceiling voltage. The influence of terminal voltage in case of thyristor type, and influence of slow response excitation system are considered. A model of fast response brushless type serves well. In most cases, $\Delta \mathrm{P}+\Delta \omega$ type PSS can be replaced by an equivalent $\Delta \mathrm{P}$ type PSS.

Proposed aggregation method is applied to test systems.

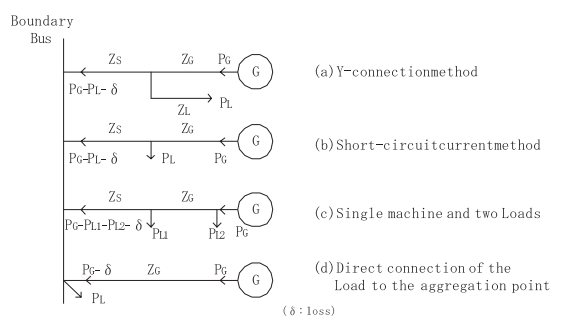

Fig. 1. Aggregation methods of power system network
Y-connection method is applied on a local system. The local system includes many hydro generators, which are assumed to have the same reactances, time constants, excitation system, and speed governor. The local system also includes many loads, which are also assumed to have the same characteristics (dynamic or static load model). The loads consume more power than the generators produce. Load branch impedance $\mathrm{Z}_{\mathrm{L}}$ includes a $6 \%$ reactance that represents the distribution system. A $3 \mathrm{LG}-\mathrm{O}$ fault cleared in 0.1 second on the double-circuit transmission line supplying to the $154 \mathrm{kV}$ substation is assumed. Calculation is performed by Transient Stability Analysis tool of CPAT. As shown in Fig. 2, the Y-connection method agrees with the detailed model very well. The example shows the need for the proposed Y-connection aggregation method in adopting a dynamic load model.

Y-connection method and generating units aggregation methods are applied to a bulk system. The resulting model reduces a bulk system, consists of complex loops, 33 generators, and 31 dynamic loads, to four generators and four dynamic loads. Aggregation accuracy is shown in Fig. 3 by weighted average angle of all generators included in the studied system. Contingency is a $3 \mathrm{LG}-\mathrm{O}$ fault on double circuit transmission line locating in midst of the system. Each load includes 50\% induction motor. Transient stability limit of detailed model is 0.06 second in fault clearing time. Stability Limit of Y-connection aggregated model is also 0.06 second in fault duration time, which is equal to that of detailed model.

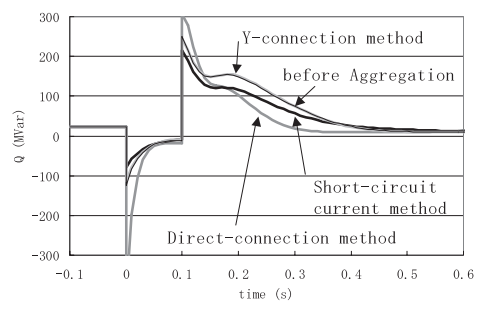

Fig. 2. Reactive power flow of A S/S $154 / 66 \mathrm{kV}$ transformer (secondary side, dynamic load)

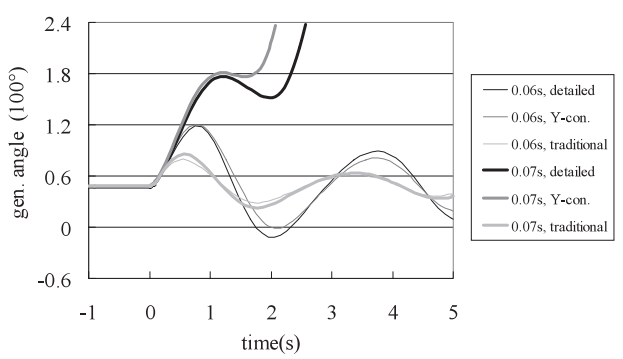

Fig. 3. Accuracy of system aggregation 


\title{
Practical Power System Aggregation Considering Dynamic Loads
}

\author{
Yoshio Yamagishi* Member \\ Shintaro Komami* Member
}

This paper presents a practical method for creating power system equivalents that can be used in power system stability analysis. The proposed method uses three branches to connect the boundary bus, generator, and load. The method is suitable to consider reactance from the boundary bus to the load for power system stability studies using a dynamic load model. Furthermore, this paper presents a practical procedure to determine the parameters of the aggregated generator, including control devices such as excitation systems, PSS (power system stabilizer), and speed governors. The quality of the various aggregation models is verified using power system stability simulations.

Keywords: power system aggregation, power system stability, dynamic load model, generating unit model, excitation system

\section{Introduction}

Power transmission networks are being operated closer to their limits. Therefore, secure operation of electric power systems increasingly requires accurate transient and dynamic stability studies. It is not practical to model an entire power system for stability analysis due to limitations, such as analysis programs, difficulties with data acquisition, and calculation speed. Therefore, it is necessary to adopt some aggregation to reduce the magnitude of the model. Research on aggregation of power system stability started in the late $60 \mathrm{~s}^{(1)}$. Despite the progress of computer power and simulation programs, the construction of an accurate, aggregated model is important issue ${ }^{(2)-(10)}$ because of increasing size and complexity of interconnected power systems. For online dynamic security assessment, aggregated models of systems are especially needed to offset computational constraints ${ }^{(2)}$.

There are two targets of aggregation: external and internal systems. The former is relatively far from the study area; thus, the linear response of the system is important. On the other hand, the internal system serves as a small portion of a study area network and testing should consider a contingency close to an aggregation point. So, the accuracy of transient and dynamic stability studies is important, and dynamic loads and the use of distributed generators affect the simulation results. The importance of proper representation of loads in power system stability studies has long been recognized. As distributed generators are placed near loads, their representation is also important. However, in aggregation methods, load representation has not been recognized important because of the focus on the aggregation of external systems.

Internal system aggregation is indispensable for the construction of models such as the IEEJ Standard models ${ }^{(3)}$ and online security assessment models of Japanese bulk power systems. Therefore, the aggregation of loads should be considered.

From the aggregation method viewpoint, aggregation

\footnotetext{
* Engineering Research \& Development Center, Hokuriku Electric Power Company

2-54, Hisakata-machi, Toyama 930-0848
}

methods can be divided broadly into two categories: (1) coherency method and (2) eigenvalue method. The former focuses on coherency of the swing curve of each generator, and is more familiar to engineers. The latter tries to preserve eigenvalues of the system, and is more precise in terms of physics and mathematics; however, it is not perfect for transient stability simulations because transient stability is nonlinear. Thus, the coherency method is more suitable for internal aggregation.

The aggregation procedure involves three steps. First, identifying coherent generators; second, aggregation of the networks; and third; the aggregation of coherent generators and their control devices.

Sufficient literature on identification methods of coherent generators is available ${ }^{(4)(5)}$. However, when such tools are not available, or identification results are not perfect, heuristic methods provide identification.

The aggregation of networks has been investigated; however, these studies usually eliminate load buses and do not consider them. There is little published information on load modeling of aggregated networks ${ }^{(6)}$. In a reduction method used for voltage instability analysis, load buses are considered, but the branches connected to the loads are eliminated ${ }^{(7)}$. Therefore, there has been no detailed research on load representation of aggregated networks, and established models do not exist.

Some literature deals with the aggregation of coherent generators and control devices. Aggregation of control systems is recognized as important issue ${ }^{(8)}$. DYNRED contains several weighted-average and least-squares frequency-domain algorithms to compute the parameters of the aggregated generator and its control models. These have limitations in aggregating highly nonlinear controls ${ }^{(9)}$. NETOMAC ${ }^{(10)}$ can consider nonlinear behavior by tuning equivalent controller parameters, such as gains and selected time constants, but users need some experiences in setting up reasonable target functions. A recent publication ${ }^{(11)}$ used non-iterative procedure and required less computation to aggregate; however, it is limited to group coherent machines equipped with the same type of control systems. 
In this paper, the authors propose a new method, named the "Y-connection method", for creating power system equivalent networks for power system stability analysis. The Yconnection method, which is a type of coherency method, has the following features:

- It uses three branches to connect the boundary bus, generator and load.

- Dynamic loads and distributed generators, which are difficult for eigenvalue methods to model, are easily modeled.

- It can take into account reactance from the boundary bus to a load and a distributed generator. The accuracy of simulations is improved by considering that reactance.

Furthermore, this paper describes a practical procedure to determine the parameters used in the coherency method. These include the aggregated generator, excitation system, PSS (power system stabilizer), and speed governor. Using this procedure, different type of control system, such as excitation system, can be aggregated satisfactory. Finally, the accuracy of the reduced models is demonstrated using power system stability simulations.

\section{Network Aggregation}

2.1 Concept of the Y-connection Method Since the aim of power system analysis or simulation is to predict any possible unstable phenomena precisely, an aggregation method should:

(1) Preserve original power flow. Power flow gives the conditions of power system stability. If the conditions are wrong, then any analysis or simulation result might be wrong.

(2) Preserve limits of voltage, transient, and dynamic stability. Since power system analysis or simulation is performed to determine practical operational limits, errors in the stability limit can be fatal. Small differences of behavior are not catastrophic.

(3) Be compatible with commercial simulation tools. Therefore, the model should consist of only the usual generators, excitation systems, speed governor, network, shunt compensation, and loads.

Figure 1(b), (c), and (d) show the structures of traditional aggregation model. Figure 1(b) shows a short-circuit current method, which is sometimes called midway load model. Short-circuit current and average angle of generators can be preserved. Figure 1(c) shows an extended model of Fig. 1(b), which has two loads to preserve the real and reactive power losses ${ }^{(12)}$. However, average angle of loads cannot be preserved in these models. Therefore, satisfaction of item (2) is doubtful for loads. Moreover, Fig. 1(d) is used as a simple model.

The authors propose a new structure for an aggregation model, shown in Fig. 1(a), named the Y-connection method. It preserves, not only short-circuit current and average angle of generators, but also the average angle of loads. In addition, it preserves the real and reactive power losses. Thus, the voltage and reactive power shown in the aggregated system are normal, and correction of the power flow is almost unnecessary. The effect of load models, including the dynamic load model, on simulation results is easily examined by just changing load representation. Moreover, as distributed generators are usually placed near loads, distributed generators

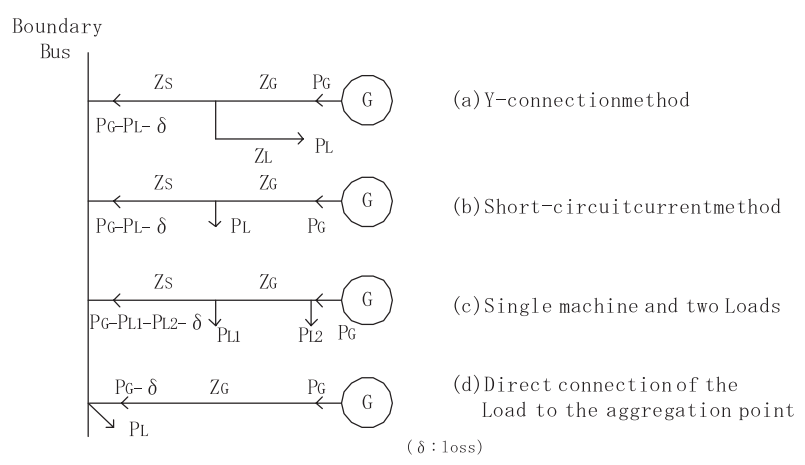

Fig. 1. Aggregation methods of power system network

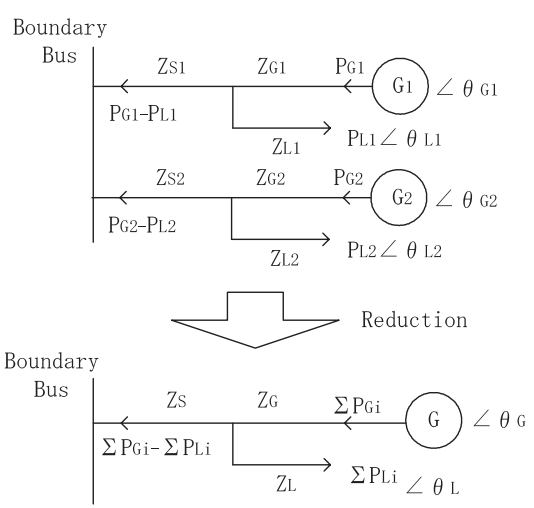

Fig. 2. Network reduction by Y-connection method

can be modeled by just connecting them to the load bus.

2.2 Aggregation Procedure In the Y-connection model three conditions are claimed.

(1) Transient impedance of the subsystem observed from trunk system is preserved, i.e., short-circuit current from the subsystem to trunk system is preserved.

(2) Output $\mathrm{kW}$-weighted average angle of generators is preserved. Therefore stability of generators may be preserved.

(3) Demand $\mathrm{kW}$-weighted average angle of loads is preserved. Therefore stability of loads may be preserved.

There are three unknown impedances and three conditions, therefore, the three impedances are calculated uniquely. In the Y-connection model, real and reactive power losses are approximately preserved. This preservation can be proven as follows. Assume that two subsystems connected to a boundary bus are aggregated to one subsystem as shown in Fig. 2.

Here, the authors define a "complex angle" $\theta$, which is defined as the summation of the product of impedance $\mathrm{Z}$ and real power flow $\mathrm{P}$, from boundary bus to the point in question as follows:

$$
\theta=\sum(Z P)
$$

Real part of $\theta$ approximately corresponds to the voltage rise or drop caused by P. Imaginary part of $\theta$ approximately corresponds to the angle of load or generator. Power flow equations related to the complex angle are written as follows:

$$
\begin{aligned}
& \theta_{G i}=Z_{S i}\left(P_{G i}-P_{L i}\right)+Z_{G i} P_{G i} \quad(i=1,2) \cdots \cdots \\
& \theta_{G}=Z_{S}\left(\sum_{i=1,2} P_{G i}-\sum_{i=1,2} P_{L i}\right)+Z_{G} \sum_{i=1,2} P_{G i} \cdots \cdots \\
& \theta_{L i}=Z_{S i}\left(P_{G i}-P_{L i}\right)-Z_{L i} P_{L i} \quad(i=1,2) \cdots \cdots
\end{aligned}
$$




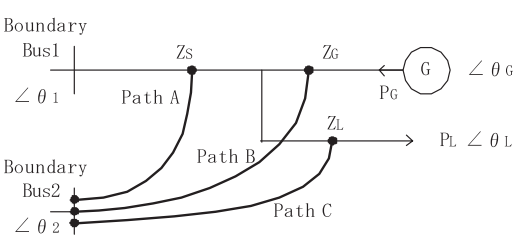

(a) Selection of path

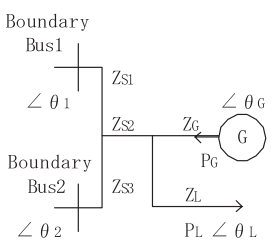

(b) Path A model

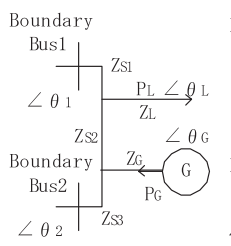

(c) Path B model

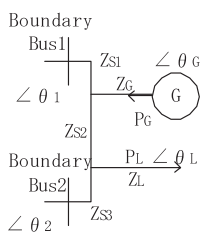

(d) Path C model
Fig. 3. Loop system with two interconnecting points

$$
\theta_{L}=Z_{S}\left(\sum_{i=1,2} P_{G i}-\sum_{i=1,2} P_{L i}\right)-Z_{L} \sum_{i=1,2} P_{L i} \cdots \cdots
$$

Claim (2) and (3) described above are written as follows:

$$
\begin{aligned}
& \theta_{G}=\sum_{i=1,2}\left(P_{G i} \theta_{G i}\right) / \sum_{i=1,2} P_{G} \\
& \theta_{L}=\sum_{i=1,2}\left(P_{L i} \theta_{L i}\right) / \sum_{i=1,2} P_{L i}
\end{aligned}
$$

From Eqs. (1) (7), following equations are obtained.

$$
\begin{gathered}
Z_{s}\left(\sum_{i=1,2} P_{G i}-\sum_{i=1,2} P_{L i}\right)^{2}+Z_{G}\left(\sum_{i=1,2} P_{G i}\right)^{2}+Z_{L}\left(\sum_{i=1,2} P_{L i}\right)^{2} \\
=\sum_{i=1,2}\left\{Z_{S i}\left(P_{G i}-P_{L i}\right)^{2}+Z_{G i} P_{G i}^{2}+Z_{L i} P_{L i}^{2}\right\} \ldots \ldots
\end{gathered}
$$

Eq. (8) indicates that real and reactive power losses by branch impedances are the same in the original system and aggregated systems. By repeating the process, any radial system can be aggregated to one generator and one load in the form shown in Fig. 2. Generators have certain internal impedance. Direct axis transient impedance $X_{d}$ ' serves for this purpose. Assuming an $\mathrm{X}_{\mathrm{d}}$ ' of $30 \%$, which is the approximate average of all generators, no detailed system data is needed, except system impedance and power flow.

2.3 Application to Loop System The Y-connection aggregation method also applies to loop system. Assume a loop system that interconnects to trunk system at two points, Bus1 and Bus2, as shown in Fig. 3. One path is chosen from path $\mathrm{A}, \mathrm{B}$, or $\mathrm{C}$. In all cases there are five unknown impedances. The choice of a path is made to preclude large negative elements in each of the impedance.

If we assume $\theta_{1}=0$ then there are six given conditions: complex phase $\theta_{\mathrm{G}}, \theta_{\mathrm{L}}$, and $\theta_{2}$, short-circuit impedance of the object system seen from Bus1 and Bus2, and the impedance of the path from Bus1 to Bus2. On the other hand, there are five unknown impedances; therefore, the given conditions cannot be fully satisfied. The choice of which condition should be sacrificed can only be decided by aggregation error through simulation.

Practically, Bus2 is often not so important for the stability of the object system. In such cases, the short-circuit
Table 1. Typical saturation curve of small generator

\begin{tabular}{|c|c|c|c|c|c|c|}
\hline $\mathrm{V}_{\mathrm{g}}$ & 0 & 0.50 & 0.80 & 1.00 & 1.10 & 1.20 \\
\hline $\mathrm{I}_{\mathrm{f}}$ & 0 & 0.50 & 0.85 & 1.15 & 1.35 & 1.75 \\
\hline
\end{tabular}

impedance seen from Bus2 or path impedance from Bus1 to Bus2 can be ignored with small aggregation error. Experimentally ignoring the short-circuit impedance seen from Bus2 is suitable.

If a loop system has 3 or more interconnections to trunk system, same procedure can be available to make aggregated network. In such case, heuristic cut-and-try procedure is needed to determine aggregated network topology. Precluding large negative elements in each of the impedance will be a guide to determine the topology.

\section{Aggregation of Generating units}

3.1 Aggregation of Generator In a real power system, each generator has unique constants, which are important because they significantly influence power system stability. As seen on many literatures, it is logical and practical to make the kVA-capability weighted values of those constants as follows and adopt them as constants of aggregated generators.

$$
\mathrm{X}_{\mathrm{d}}, \mathrm{X}_{\mathrm{d}}{ }^{\prime}, \mathrm{X}_{\mathrm{d}}{ }^{\prime}, \mathrm{T}_{\mathrm{d}}, \mathrm{T}_{\mathrm{d}}{ }^{\prime}, \mathrm{X}_{\mathrm{q}}, \mathrm{X}_{\mathrm{q}}{ }^{\prime}, \mathrm{X}_{\mathrm{q}}{ }^{\prime}, \mathrm{T}_{\mathrm{q}}{ }^{\prime}, \mathrm{T}_{\mathrm{q}}{ }^{\prime}, \mathrm{X}_{\mathrm{l}}, \mathrm{T}_{\mathrm{a}}
$$

Saturation is also important. If saturation is significant, then the positive and negative effects of fast excitation will be somewhat lost. Usually, the saturation characteristic is represented by no load excitation current in per unit value when terminal voltage is $0,0.5,0.8,1.0,1.1$, and 1.2 per unit. However, there are many generators without detailed data. Central Research Institute of the Electric Power Industry (CRIEPI) had investigated the reactances and time constants of many thermal and hydro generators, and provided standard constants in Transient Stability Analysis tool of CPAT (CRIEPI's Power System Analysis Tools). In addition, the authors introduce a typical saturation curve induced from measurements taken on a number of small generators (Table 1).

3.2 Aggregation of Exciter Constants of excitation systems are more important than those of generators, because power system transient and dynamic stability depend on excitation system design. Among various factors, the two most important factors are, frequency response, which influences dynamic stability, and forcing ability, which influences transient stability. In addition, PSS design stated is critical for dynamic stability.

If there is only one type of excitation system block diagram in the aggregation target, then that diagram is appropriate as the aggregated excitation system, and some tools have been proposed to aggregate these system ${ }^{(11)}$. However, when different kinds of excitation systems are found in aggregation target, it is difficult to choose the block diagram of the aggregated excitation system. In such cases, practically the largest generator's parameters are used as the aggregated system. The authors propose to use a general exciter model, and to determine the parameters by following procedure. Figure 4(a) shows a sample of a general exciter block diagram.

It is difficult to generate an equivalent weighted average for frequency response-some clues are needed. There are 


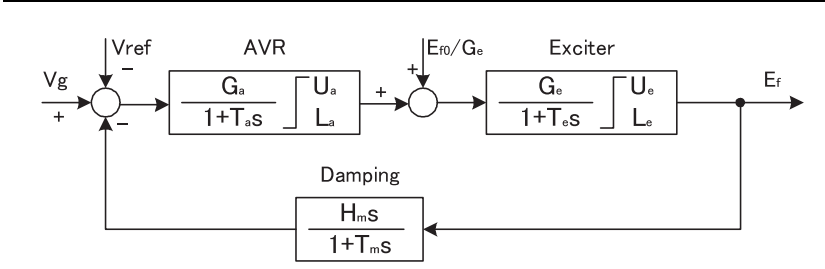

(a) General type

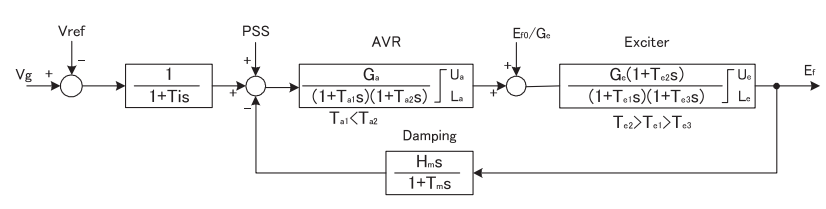

(b) AC exciter type

Fig. 4. Block diagrams of excitation systems

four factors that best describe the frequency response of an excitation system; therefore, it is logical to incorporate generator capability weighted values as factors of the aggregated excitation system. Equations are attached as a sample of the AC exciter model shown in Fig. 4(b).

(1) Middle frequency feedback gain: $F$

$$
\mathrm{F}=1+\mathrm{G}_{\mathrm{a}} \mathrm{G}_{\mathrm{e}}\left(\mathrm{T}_{\mathrm{e} 2} / \mathrm{T}_{\mathrm{e} 1}\right)\left(\mathrm{H}_{\mathrm{m}} / \mathrm{T}_{\mathrm{m}}\right)
$$

(2) Middle frequency gain: $G_{M}$

$$
\mathrm{G}_{\mathrm{M}}=\mathrm{G}_{\mathrm{a}} \mathrm{G}_{\mathrm{e}}\left(\mathrm{T}_{\mathrm{e} 2} / \mathrm{T}_{\mathrm{e} 1}\right) / \mathrm{F}
$$

(3) Middle frequency turnover time constant: $T_{m}$

(4) High frequency roll-off time constant: $T_{H}$

$$
T_{\mathrm{H}}=T_{\mathrm{i}}+\left(T_{\mathrm{a} 1}+T_{\mathrm{a} 2}+T_{\mathrm{e} 3}\right) / \mathrm{F}
$$

Low frequency gain, $\mathrm{G}_{\mathrm{L}}$, of the aggregated generator is consequently $G_{L}=F_{M}$. Usually frequency response derived by the method stated above does not agree with the weighted average of original excitation systems, therefore, some correction is needed. The authors made the correction by cut-and-try by observing frequency response of average and aggregated system on spreadsheet program. User may need some experience to fit the frequency response. Future work is required to improve the efficiency of proposed method.

Three additional factors best describe the forcing ability of excitation system, thus we adopt generator capability weighted values of them as factors of aggregated generator.

(5) Rise time: $T_{r}$

$$
\mathrm{T}_{\mathrm{r}}=\mathrm{T}_{\mathrm{e} 3} /\left(\mathrm{U}_{\mathrm{a}} \mathrm{G}_{\mathrm{e}} \mathrm{T}_{\mathrm{e} 2} / \mathrm{T}_{\mathrm{e} 1}\right)
$$

(6) Descend time: $T_{d}$

$$
\mathrm{T}_{\mathrm{d}}=\mathrm{T}_{\mathrm{e} 3} /\left(-\mathrm{L}_{\mathrm{a}} \mathrm{G}_{\mathrm{e}} \mathrm{T}_{\mathrm{e} 2} / \mathrm{T}_{\mathrm{e} 1}\right)
$$

(7) Ceiling voltage: $U_{e}$ and $L_{e}$

In case of thyristor type excitation systems, the exciter time constant $T_{e}$, rise time $T_{r}$, and descend time $T_{d}$ are nearly equal zero. If some thyristor type excitation systems, shown in Fig. 5(a), are included in the aggregated generator, then $U_{a}$ is sometimes calculated as less than $\mathrm{U}_{\mathrm{e}}-\mathrm{E}_{\mathrm{f} 0}\left(\mathrm{E}_{\mathrm{f} 0}\right.$ means initial field voltage), which means a shortage of ceiling voltage. In such cases, representation by fast-response brushless type shown in Fig. 5(b) is suitable. The ratio of DC feedback $\beta$ is

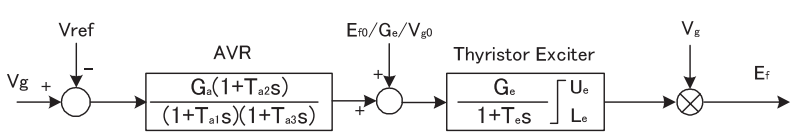

(a) Thyristor type

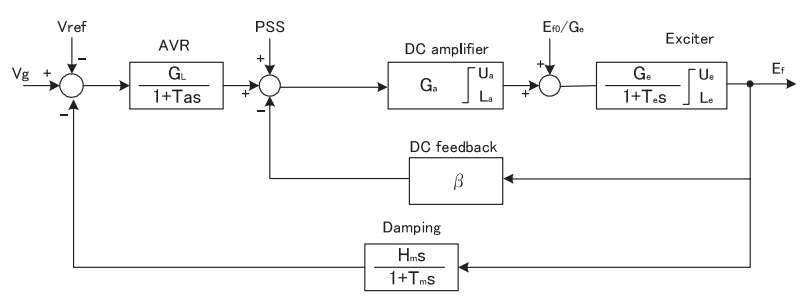

(b) Brushless type

Fig. 5. Block diagrams of excitation systems

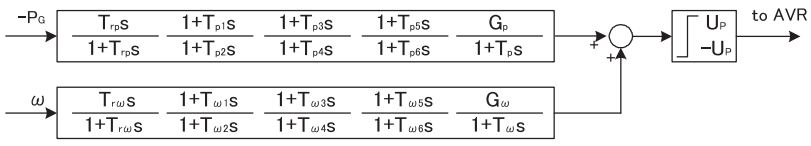

Fig. 6. Block diagram of $\Delta \mathrm{P}+\Delta \omega$ type PSS

set as follows:

$$
\beta=(\mathrm{A}-1) /\left(\mathrm{AG}_{\mathrm{e}}\right)
$$

Therefore, the minor feedback gain is $\mathrm{F}=1+\mathrm{AG}_{\mathrm{e}} \beta=A$, closed loop gain is $\mathrm{G}_{\mathrm{e}}$, and the equivalent exciter-time constant is $\mathrm{T}_{\mathrm{e}}{ }^{\prime}=\mathrm{T}_{\mathrm{e}} / \mathrm{F}$.

In case of major contingency in a trunk system, each excitation system cannot always reach its ceiling voltage for various reasons.

- In case of a thyristor type, the ceiling voltage is proportional to generator's terminal voltage, which takes a smaller value than nominal when the rotor angle is leading during the power swing. Therefore, this type of ceiling voltage must be discounted to $\mathrm{KU}_{\mathrm{e}}(\mathrm{K}<1)$. $\mathrm{K}$ has been experimentally proven to be about 0.9 at near stability limit. If the aggregated excitation system is represented as thyristor type, then this discounting is not needed.

- Slow response excitation system cannot reach its ceiling voltage at the first swing peak, that is, the forcing ability is limited by response rather than by the ceiling voltage itself. Through case studies, effective ceiling voltage $\mathrm{U}_{\mathrm{e}}$, is proved to be as follows:

$\mathrm{U}_{\mathrm{e}}{ }^{\prime}=\min \left\{\mathrm{U}_{\mathrm{e}}, \mathrm{E}_{\mathrm{f} 0}+\mathrm{T}_{\mathrm{u}} / \mathrm{T}_{\mathrm{r}}\right\}$

Here $T_{u}$ stands for the time allowed for excitation system to raise its ceiling voltage. Through case studies, $T_{u}$ has been determined to be about 0.5 . $\mathrm{E}_{\mathrm{f} 0}$ is about 2.0 in thermal generators that usually operate at maximum load, and 1.3 in hydro generators that usually operate at partial load.

3.3 Aggregation of PSS Damping performance of generator is mainly affected by PSS. Similar to excitation systems, weighted average of frequency response and ceiling voltage must be preserved, considering generators without PSS. Two types of PSS are used: $\Delta P$ type, which is effective at higher frequencies, and $\Delta \omega$ type, which is effective in lower frequencies. A block diagram of $\Delta P+\Delta \omega$ type PSS is shown in Fig. 6. Sometimes series double-reset filters (lowpass filter) are adopted. $T_{p 1}$ to $T_{p 6}$ and $T_{\omega 1}$ to $T_{\omega 6}$ are used for phase-angle correction. 
There is a relationship between vector $\Delta P_{g}$ and vector $\Delta \omega$ as follows:

$$
\Delta \omega=\frac{-\Delta P g}{M s+D}
$$

Therefore, the vector gain of $\Delta P$ type $G_{p}$ and that of $\Delta \omega$ type $G_{\omega}$ are combined to another vector gain of $\Delta P$ type $G_{p}^{*}$ as follows:

$$
G p^{*}=G p+\frac{G \omega}{M s+D}
$$

$M$ is the unit inertia constant of the generator. $D$ is the generator damping. $D$ is in proportion to four times powered swing frequency: $\mathrm{fs}^{4}$. It is well known that $\Delta \omega$ type PSS is effective in low frequency power swings, where the value of $D$ is small, so $D$ can be neglected. Therefore, a $\Delta P+\Delta \omega$ type PSS can be represented by an equivalent $\Delta P$ type PSS. From Eq. (16), it is understood that $\Delta \omega$ has about 90 degreelagged angle compared to $-\Delta P_{g}$. Therefore, $G_{p}^{*}$ has a large lagging angle, and has no less than one large time constant in its denominator.

3.4 Aggregation of Speed Governor For several seconds after a fault, speed governors may have some effect on generators. The most important factor is droop $\delta$, which is the necessary frequency change to make $100 \%$ output change. In a thermal generator, the speed governor affects only the high-pressure turbine. Therefore, weighted average of equivalent governor gain $\mathrm{G}_{\mathrm{gov}}$ should be preserved. Here, $K_{\mathrm{HP}}$ is the output ratio of the high-pressure turbine. $\delta$ should be regarded as infinity in case of load-limiter operation and for hydro generator, whose governor is slow as compared with thermal generators.

$$
\mathrm{G}_{\mathrm{gov}}=\mathrm{K}_{\mathrm{HP}} / \delta
$$

Rarely, special considerations are required:

- The output of a low-pressure turbine is affected by intercept valve operation, in case of a single governor on a thermal generator. The phenomenon improves transient stability. Governor constants of the dominant generator should be chosen, and the droop $\delta$ should be tuned by Eq. (18).

- The speed governor of a hydro generator has large angle lagging because of water hammering, which spoils the dynamic stability and frequency stability of the islanded system. If a hydro generator is dominant, then the aggregated governor should be modeled as the average for hydro governors.

\section{Application}

4.1 Application to Local System The difference between traditional and the proposed Y-connection method is only in structure. If the generators and loads included in the original model have the same characteristics, then the errors between original and aggregated models might be caused by the structure. Therefore, a local system $(66 \mathrm{kV}$ system of a $154 \mathrm{kV}$ substation secondary) is chosen as the example (Fig. 7).

The local system includes many hydro generators, which are assumed to have the same reactances, time constants, excitation system, and speed governor. The local system also

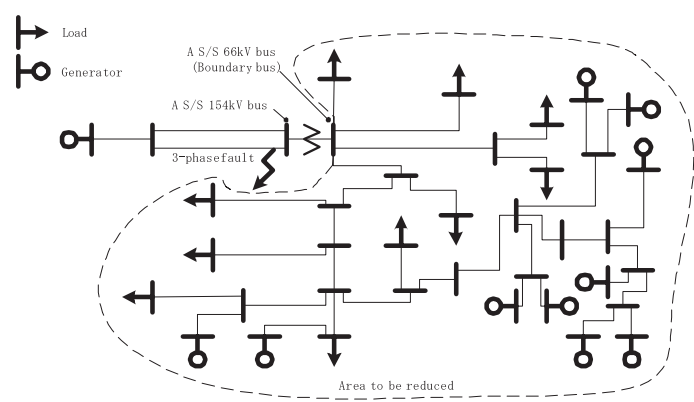

Fig. 7. One-line diagram of the test system (local system)

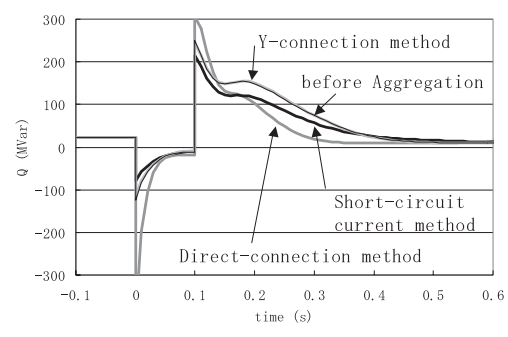

(a) Dynamic load model

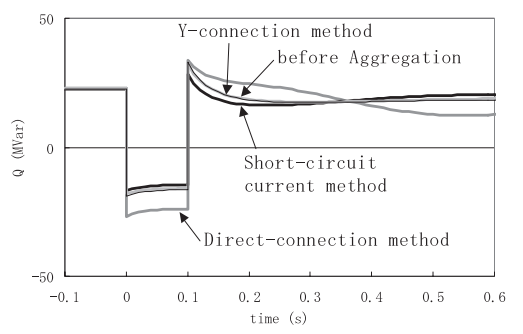

(b) Static load model

Fig. 8. Reactive power flow of A S/S $154 / 66 \mathrm{kV}$ transformer (secondary side)

includes many loads, which are also assumed to have the same characteristics. Two kinds of load characteristics are assumed.

(a) Dynamic load model (50\% induction motor and $50 \%$ constant admittance)

(b) Static load model (constant current)

The loads consume more power than the generators produce. Load branch impedance $\mathrm{Z}_{\mathrm{L}}$ includes a $6 \%$ reactance that represents the distribution system. A 3LG-O fault cleared in 0.1 second on the double-circuit transmission line supplying to the $154 \mathrm{kV}$ substation is assumed. Calculation is performed by Transient Stability Analysis tool of CPAT.

Aggregation accuracy is shown in Fig. 8. The Yconnection method agrees with the detailed model very well both in the dynamic load model case and static load model case, whereas traditional methods (Short-circuit current method and Direct -connection method) show significant error. The authors have also calculated the single machine and two loads model shown in Fig. 1(c), but it is thought that the model is not suitable for this case, because $\mathrm{P}_{\mathrm{L} 2}$ has a considerable negative value.

In the dynamic load case, fluctuations of reactive power flow during and after the fault is relatively sharp, so the effect of load have great influence upon the simulation results. Therefore, proposed Y-connection aggregation method is 


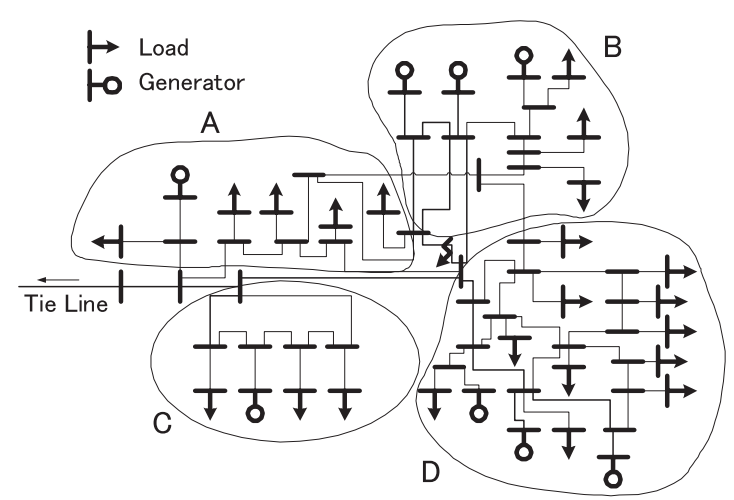

Fig. 9. One-line diagram of the test system (large system)

effective to the dynamic load model.

4.2 Application to Large System Usually aggregation is applied to local and external systems. As these systems are electrically far from the studied trunk system, have strong coherency, and have small effect on stability, their aggregation error should be negligible. In this section, the authors apply the internal system aggregation, which is more difficult than external system aggregation, to the trunk system. This should clearly prove the accuracy or limitations of the method, even in transient stability, which is thought to be the most difficult phenomenon for aggregation.

Major structure of the test system is shown in Fig. 9. Test system is located at one end of gigantic interconnection, consists of 33 generators and 31 loads, forms a complex loop system, interconnects to the outer system by one tie line, sends a little power to outer system, and has strong coherency against outer system.

The object system (Fig. 9) is aggregated to four subsystems: A, B, C, and D. Groups of subsystems are selected by following rules.

(a) To preserve trunk network topology, keep major loop system unchanged.

(b) To minimize the number of boundary bus of each subsystem, generator groups and boundary buses are empirically determined without using coherent generators identification tools.

To aggregate the loop system, path B in Fig. 3 proved suitable. Each subsystem consists of one generator and one load. The governor model has no special corrections, as stated in section 3.4. The resulting model reduces a bulk system, consists of complex loops, 33 generators, and 31 dynamic loads, to four generators and four dynamic loads (Fig. 10). Traditional (short-circuit current method) aggregated model is used as a contrast case. Traditional model does not have load branches shown in Fig. 10 as thick dashed lines.

Aggregation accuracy is shown in Fig. 11 by the weighted average angle of all generators included in the studied system. The contingency is a 3LG-O fault on the doublecircuit transmission line located in the middle of the system. Each load includes a 50\% induction motor. The transient stability limit of the detailed model is 0.06 seconds (faultclearing time). The stability limit of Y-connection aggregated model is also 0.06 seconds. Transient stability limit of traditional aggregated model is 0.13 seconds, which is much more optimistic than the detailed or Y-connection model. The

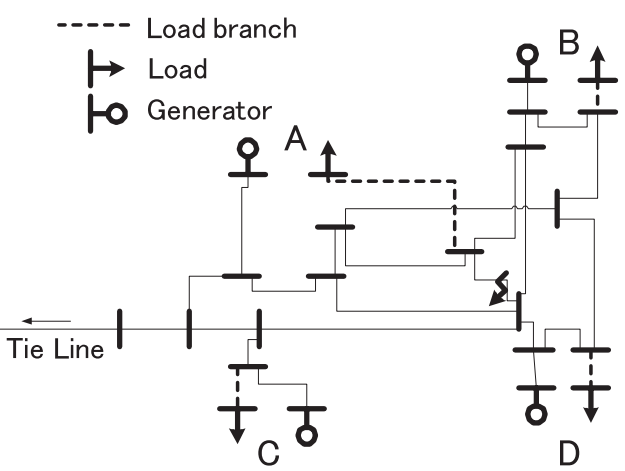

Fig. 10. Aggregated system

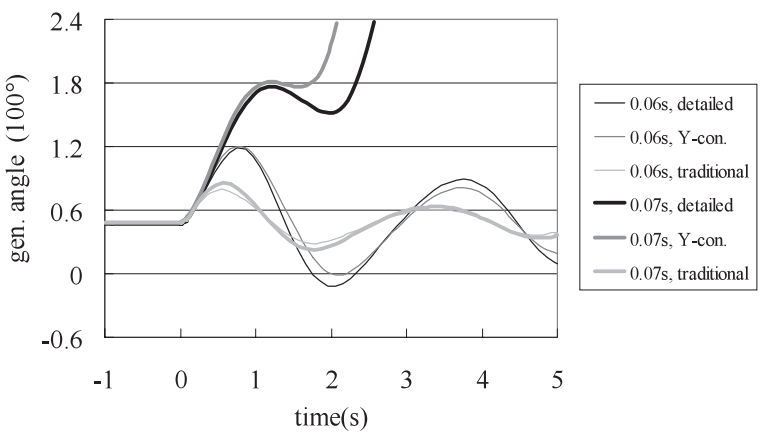

Fig. 11. Accuracy of system aggregation

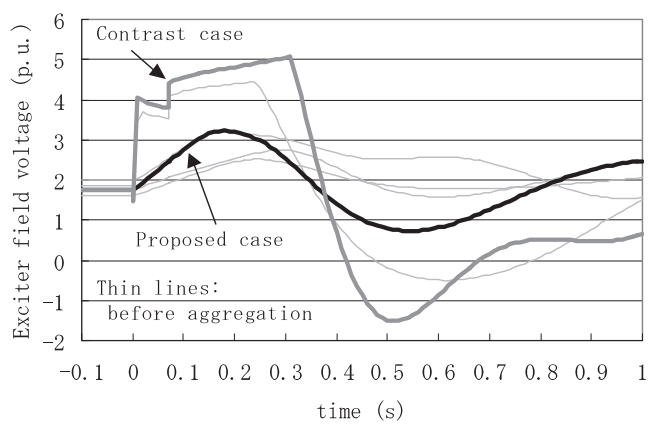

Fig. 12. Field voltage of generators

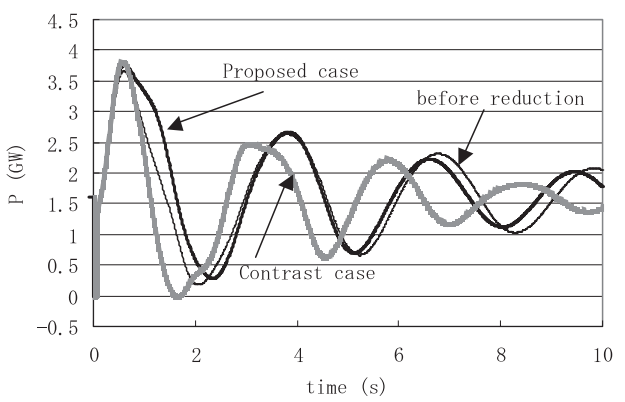

Fig. 13. Tie-line active power flow

traditional aggregation method evaluates transient stability optimistically, and can overlook existing risk. Practically, it is misleading. This poor accuracy arises from neglecting load-branch impedance. Therefore Y-connection method including load branch impedance is indispensable for aggregation in case of considering dynamic load.

\subsection{Effect of Excitation System Aggregation}

Quality of the proposed generating units aggregation method is also checked by the system introduced in 
section 4.2. Two cases of excitation system aggregation are examined: (1) the proposed model stated in section 3.2, and (2) the contrasting model that uses the largest generator's parameters in each subsystem. Same network data aggregated by the Y-connection method is used in both cases.

As shown in Figs. 12 and 13, proposed model agrees closely with the detailed model. Generally, as the largest generator has the modern style of excitation system that has small time constants, generators of contrast case respond too fast.

\section{Conclusion}

This paper proposed a practical aggregation method for power system stability analysis. The key concepts of the method are as follows:

(1) The proposed aggregation method is to aggregate network, generators, saturation characteristics of generators, exciting systems, PSSs, and speed governors independently, and then synthesizes them into one aggregated system. Therefore, the method is practical and realistic in execution; however, its accuracy must be carefully examined.

(2) For aggregating a network, the Y-connection method is adopted. The weighted average complex angle of generators and loads are preserved. Consequently, it is demonstrated that real and reactive power losses are approximately preserved, and it requires no correction of power flow at the initial condition. The Y-connection method assumes that stability of generators and loads is evaluated best by angle. The traditional aggregation method preserves only the average angle of generators, therefore, cannot evaluate stability precisely.

(3) In aggregating the excitation system, the weighted average of frequency response and forcing ability are preserved. Forcing ability is evaluated by rise time and ceiling voltage. The influence of terminal voltage in case of thyristor type, and influence of slow response excitation system are considered. A model of fast response brushless type serves well. In most cases, $\Delta \mathrm{P}+\Delta \omega$ type PSS can be replaced by an equivalent $\Delta \mathrm{P}$ type PSS.

The proposed aggregation method is applied to test systems. Two examples, difficult test cases for aggregation, are adopted to test the proposed method. The proposed method shows good accuracy, whereas the traditional method proves to be much more optimistic. The traditional method can overlook existing risk and is practically misleading, in case of using the dynamic load model. Accuracy of proposed excitation aggregation method is also verified.

This method includes some heuristic cut-and-try procedures to determine the parameters. Future work is required to improve the efficiency of proposed method.

(Manuscript received Aug. 25, 2006, revised July 3, 2007)

\section{References}

( 1 ) H.E. Brown, R.B. Shipley, D. Coleman, and R.E. Nied, Jr: "A Study of Stability Equivalents", IEEE Trans. Power Apparatus Syst., Vol.PAS-88, No.3, pp.200-207 (1969)

( 2 ) M. Saeki, H. Matsuoka, Y. Nagae, M. Tanaka, K. Omata, and M. Sato: "Development and application of a new approach to construct external equivalents for power system stabilizing controller", T. IEE Japan, Vol.120-B, No.4, pp.537-544 (2000) (in Japanese)

( 3 ) IEEJ Technical Committee: "Power System Standard Model", IEEJ Technical Report, No.754 (1999) (in Japanese)

( 4 ) L. Wang, M. Klein, S. Yirga, and P. Kundur: "Dynamic Reduction of Large Power Systems for Stability Studies”, IEEE Trans. Power Syst., Vol.12, No.2, pp.889-895 (1997)

( 5 ) W. Price, J. Chow, A. Hargrave, B. Hurysz, and P. Hirsch: "Large-Scale System Testing of a Power System Dynamic Equivalencing Program", IEEE Trans. Power Syst., Vol.13, No.3, pp.768-774 (1998)

( 6 ) Y. Yamagishi, T. Ueda, and S. Komami: "Power System Reduction Including Dynamic Load", The Papers of Technical Meeting on Power Engineering and Power System Engineering, IEE Japan, PE-05-41, PSE-05-48 (2005-9) (in Japanese)

( 7 ) J. McCalley, J. Dorsey, J. Luini, R. Mackin, and G. Molina: "Subtransmission Reduction for Voltage Instability Analysis", IEEE Trans. Appli. Superconduct., Vol.3, No.1, pp.349-356 (1993)

( 8 ) S. Joo, C. Liu, L. Jones, and J. Choe: "Coherency and Aggregation Techniques Incorporating Rotor and Voltage Dynamics", IEEE Trans. Power Syst., Vol.19, No.2, pp.1068-1075 (2004)

( 9 ) G. Jang, B. Lee, S. Kwon, H. Kim, Y. Yoon, and J. Choo: "Development of KEPCO equivalent systems for the KEPCO enhanced power system simulator", Electrical Power \& Energy Systems, Vol.23, pp.577-583 (2001)

(10) X. Lei, D. Povh, and O. Ruhle: "Industrial approaches for dynamic equivalents of large power systems", Proc. Power Eng. Soc. Winter Meeting, Vol.2, pp.1036-1042 (2002)

(11) M. Ourari, L. Dessaint, and V. Do: "Dynamic Equivalent Modeling of Large Power Systems Using Structure Preservation Technique", IEEE Trans. Power Syst., Vol.21, No.3, pp.1284-1295 (2006)

(12) S. Hosoki, K. Tanaka, and H. Yanagida: "A Study on Various Order Reduction Method of Power System", Proc. of the Tenth Annual Conference of Power \& Energy Society, IEE Japan, No.236 (1999-8) (in Japanese)

Yoshio Yamagishi (Member) He was born on Aug. 25, 1968. He re-

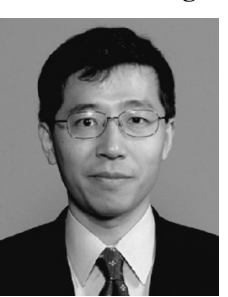
ceived BS and MS from the University of Tokyo in 1991 and 1993, respectively. He joined Hokuriku Electric Power Company in 1993, and is currently a research engineer in the Engineering \& Environmental Technology Development Center of Hokuriku EPCo. He is a member of IEEE.

Shintaro Komami (Member) He was born on Sep. 13, 1952. He

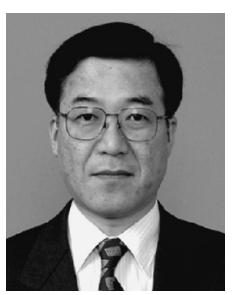
received BS from the University of Tokyo in 1975. He joined Hokuriku Electric Power Company in 1975. He is a general manager of the Engineering \& Environmental Technology Development Center of Hokuriku EPCo. 